TRANSACTIONS OF THE

AMERICAN MATHEMATICAL SOCIETY

Volume 195, 1974

\title{
ON A CERTAIN SUM IN NUMBER THEORY. II
}

BY

\section{BŘETISL AV NOVÁK( (1)}

ABSTRACT. We derive "exact order" of the function

$$
\sum_{k \leq \sqrt{x}} k^{\rho} \min ^{\beta}\left(\frac{\sqrt{x}}{k}, \frac{1}{P_{k}}\right)
$$

Here $\rho$ and $\beta$ are nonnegative real numbers and, for given real $\delta_{1}, \delta_{2}, \cdots, \delta_{\gamma}$, $P_{k}=\max _{j}\left\langle k \delta_{j}\right\rangle$ where $\langle t\rangle$, for real $t$, denotes distance of $t$ from the nearest integer. Using our results, we obtain the solution of the basic problem in the theory of lattice points with weight in rational many-dimensional ellip soids.

I. Introduction. Let $Q(u)=Q\left(u_{1}, u_{2}, \ldots, u_{r}\right)$ be a positive definite quadratic form in $r \geq 2$ variables with a symmetric integral coefficient matrix and determinant $D$. Let $b_{j}, M_{j}$ be integers, $M_{j}>0$ and $a_{j}$ be real numbers, $j=$ $1,2, \cdots$, r. Let us put, for $x>0$,

$$
A(x)=\sum \exp \left(2 \pi i \sum_{j=1}^{r} a_{j} u_{j}\right), \quad V(x)=\frac{\pi^{r / 2} x^{r / 2} \exp \left(2 \pi i \sum_{j=1}^{r} a_{j} b_{j}\right)}{\sqrt{D} M_{1} M_{2} \cdots M_{r} \Gamma(r / 2+1)} \delta,
$$

where $\delta=1$ if all the $a_{j} M_{j}$ are integers, and $\delta=0$ otherwise. Here the summation runs over all systems $u=\left(u_{1}, u_{2}, \ldots, u_{r}\right)$ of real numbers such that $Q(u) \leq x$ and $u_{j} \equiv b_{j}\left(\bmod M_{j}\right), j=1,2, \cdots, r$.

For $\rho \geq 0$ put $P_{0}(x)=P(x)=A(x)-V(x)$ and

$$
\begin{gathered}
P_{\rho}(x)=\frac{1}{\Gamma(\rho)} \int_{0}^{x} P(t)(x-t)^{\rho-1} d t \text { for } \rho>0, \\
M_{\rho}(x)=\int_{0}^{x}\left|P_{\rho}(t)\right|^{2} d t \text { for } \rho \geq 0 .
\end{gathered}
$$

In the papers [3], [5] and [9] it is shown that O-estimates of these functions can be obtained from 0 -estimates of the functions

Received by the editors October 30, 1972

AMS (MOS) subject classifications (1970). Primary 10F99; Secondary 10J25.

Key words and phrases. Lattice points with weight in ellipsoids, simultaneous approximation.

(1) The author wrote this paper during his stay at the University of Illinois, Urbana 


$$
\begin{aligned}
& F_{1}(x)=\sum_{k \leq \sqrt{x}} \lg 2 k \min ^{r / 2-1}\left(\sqrt{x} / k, 1 / P_{k}\right), \\
& F(x)=F_{\rho, \beta}\left(x ; a_{j}\right)=\sum_{k \leq \sqrt{x}} k^{\rho} \min ^{\beta}\left(\sqrt{x} / k, 1 / P_{k}\right) .
\end{aligned}
$$

Here, $\rho$ and $\beta$ are nonnegative real numbers, $k$ is a positive integer and $P_{k}=$ $\max _{j=1,2, \cdots, r}\left\langle\alpha_{j} M_{j} k\right\rangle$, where $\langle t\rangle$, for real $t$, denotes the distance of $t$ from the nearest integer, and where we put $\min (A, 1 / B)=A$ for $B=0$.

In fact, we have proved in the aforementioned papers that

$$
\begin{aligned}
& P(x) \ll x^{r / 4-1 / 2} F_{1}(x) \text { for } r>4, \\
& P_{\rho}(x) \ll x^{r / 4-1 / 2} F_{\rho, r / 2-1}(x) \text { for } r>4 \text { and } \rho>0
\end{aligned}
$$

and

$$
M_{\rho}(x) \ll x^{r / 2-1 / 2} F_{2 \rho+1, r-1}(x)
$$

Here, we write $A \ll B$ instead of $|A| \leq c B$, where $c$ is a constant depending only on $a_{j}, M_{j}, b_{j}, \rho, \gamma, \epsilon$ and $\beta$. In the sequel we always assume that $x>c$ and $\rho \geq 0, \beta \geq 0$.

In [6], considered as the first part of this paper, the function $F(x)$ is studied especially in the case when $a_{1}=a_{2}=\cdots=a_{i}$. Let us recall some results (see $[6$, Theorems $1,5,6$ and 7]).

(A) Always,

$$
\begin{array}{ll}
x^{(\rho+1) / 2} \ll F(x) \ll x^{\beta / 2} & \text { for } \rho<\beta-1, \\
x^{(\rho+1) / 2} \ll F(x) \ll x^{\beta / 2} \lg x & \text { for } \rho=\beta-1, \\
x^{(\rho+1) / 2} \ll F(x) \ll x^{(\rho+1) / 2} & \text { for } \rho>\beta-1,
\end{array}
$$

and these results generally cannot be improved.

(B) Let $\rho<\beta-1, \gamma>0$, and let the inequality $P_{k} \ll k^{-\gamma}$ be fulfilled for infinitely many $k$. Then $F(x)=\Omega\left(x^{f}\right)$, where

$$
f=(\beta \gamma+\rho) / 2(\gamma+1) \text {. }
$$

(C) Let $\rho\langle\beta-1, \gamma\rangle 0$, and let the inequality $P_{k} \gg k^{-\gamma}$ hold for all $k_{\text {o }}$ Then

$$
F(x) \ll x^{f+1 / 2(\gamma+1)}
$$

If moreover $a_{1}=a_{2}=\cdots=a_{r}$, then 


$$
\begin{array}{ll}
F(x) \ll x^{f} & \text { for } \rho<\beta-2, \\
F(x) \ll x^{f}+x^{(\rho+1) / 2} \lg x & \text { for } \rho=\beta-2, \\
F(x) \ll x^{f}+x^{(\rho+1) / 2} & \text { for } \rho>\beta-2 .
\end{array}
$$

The aim of the present paper is to improve the relation (7), actually we are going to prove the following

Main Theorem. Let $\rho\langle\beta-1, \gamma\rangle 0$ and let the inequality $P_{k} \gg k^{-\gamma}$ bold for all k. Then $F(x) \ll x^{f}$ for $\rho<\beta-1-1 / \gamma$, and $F(x) \ll x^{(\rho+1) / 2} \lg _{g}^{\epsilon} x$ other. wise, where we put $\epsilon=1$ for $\rho=\beta-1-1 / \gamma$ or $\beta \gamma=1$, and $\epsilon=0$ otherwise.

II. Proof of the Main Theorem. Let, for $n=0,1, \ldots$,

$$
T_{n}=\sum k^{\rho} \min ^{\beta}\left(\frac{\sqrt{x}}{k}, \frac{1}{P_{k}}\right)
$$

where we are summing over all $k, 2^{n} \leq k<2^{n+1}$. Thus

$$
F(x) \ll \sum_{2^{n} \leq \sqrt{x}} T_{n} .
$$

First we need some estimates of the number of $k$, for which $P_{k}$ is small. Let $l$ and $M$ be positive integers, and let $M \leq k_{1}<k_{2}<\ldots<k_{\nu} \leq 2 M$ be numbers of the interval $[M, 2 M]$ fulfilling the inequality $2^{-l-1} \leq P_{k}<2^{-l}$. Denote by $K$ the smallest positive integer such that $P_{k}<2 \cdot 2^{-l}$. From the obvious inequality $\left\langle\xi_{1} \pm \xi_{2}\right\rangle \leq\left\langle\xi_{1}\right\rangle+\left\langle\xi_{1}\right\rangle, \xi_{1}$ and $\xi_{2}$ are real, we obtain $k_{1} \geq K, k_{2}-$ $k_{1} \geq K, \cdots, k_{\nu}-k_{\nu-1} \geq K$, and then $k_{\nu} \geq \nu K$. Hence, using the assumption $2 \cdot 2^{-l}>P_{K} \gg K^{-\gamma} \geq\left(\nu / k_{\nu}\right)^{\gamma} \geq(\nu / 2 M)^{\gamma}$, we conclude that

$$
\nu \ll 2^{-l / \gamma} M \text {. }
$$

(A similar method was used in the paper [2, p. 216], the same method in the paper [1, p. 131]). We are going to use this result in the sequel without any further reference. Further, let us note that

$$
\sum_{k \leq \sqrt{x} ; P_{k}>c} k^{\rho} \min ^{\beta}\left(\sqrt{x} / k, 1 / P_{k}\right) \ll \sum_{k<\sqrt{x}} k^{\rho} \ll x^{(\rho+1) / 2} .
$$

Thus (cf. the assertion (A) of Introduction) in $T_{n}$ we can sum only over those $k$ with $P_{k}<c$. Finally let us recall that it is sufficient to consider only these $l$, with $2^{l} \ll M^{\gamma}$ (see (9)).

Now we successively obtain 


$$
\begin{aligned}
T_{n} & \ll \sum_{2^{l} \ll 2^{\gamma / n}} 2^{n} 2^{-l / \gamma} 2^{n(\rho-\beta)} \min ^{\beta}\left(\sqrt{x}, 2^{n+l}\right) \\
& =2^{n(\rho-\beta+1)} \sum_{2^{l}<\cdot 2^{\gamma n}} 2^{-l / \gamma} \min ^{\beta}\left(\sqrt{x}, 2^{n+l}\right) .
\end{aligned}
$$

Let us consider two cases. First, let $2^{n(\gamma+1)} \ll \sqrt{x}$. Hence $2^{n+l} \ll \sqrt{x}$ provided $2^{l} \ll 2^{\gamma_{n}}$. In this case we obtain

$$
T_{n} \ll 2^{n(\rho-\beta+1)} \sum_{2^{l} \ll 2^{\gamma / n}} 2^{-l / \gamma+\beta(n+l)}=2^{n(\rho+1)} \sum_{2^{l} \ll 2^{\gamma_{n}}} 2^{l(\beta-1 / \gamma)} .
$$

Thus

$$
T_{n} \ll \begin{cases}2^{n(\gamma \beta+\rho)} & \text { for } \beta y>1 \\ 2^{n(\rho+1)} n & \text { for } \beta y=1 \\ 2^{n(\rho+1)} & \text { for } \beta \gamma<1\end{cases}
$$

Summing over all $n$ with $2^{n(\gamma+1)} \ll \sqrt{x}$, i.e., $2^{n} \ll x^{1 / 2(\gamma+1)}$ we obtain

$$
\sum_{2^{n}<x^{1 / 2(\gamma+1)}} T_{n} \ll \begin{cases}x^{f} & \text { for } \beta y>1, \\ x^{f} \lg x & \text { for } \beta y=1, \\ x^{(p+1) / 2(\gamma+1)} & \text { for } \beta y<1 .\end{cases}
$$

In the sequel we may suppose that $2^{n(\gamma+1)} \gg \sqrt{x}$ and let us recall that $2^{n} \ll \sqrt{x}$. We obtain

$$
\begin{aligned}
& T_{n} \ll 2^{n(p-\beta+1)} \sum_{2^{l} \ll 2^{\gamma / n}} 2^{-l / \gamma} \min ^{\beta}\left(\sqrt{x}, 2^{n+l}\right) \\
& \ll 2^{n(\rho-\beta+1)}\left\{2_{2^{n \beta}} \sum_{2^{l+n}<2^{n(\gamma+1)} ; 2^{l+n} \leq \sqrt{x}} 2^{l(\beta-1 / \gamma)}+x^{\beta / 2} \sum_{2^{l}>\sqrt{x} / 2^{n}} 2^{-l / \gamma}\right\} \\
& \ll 2^{n(\rho+1)} \sum_{2^{l} \leq \sqrt{x} / 2^{n}} 2^{l(\beta-1 / \gamma)}+x^{(\beta-1 / \gamma) / 2} 2^{n(\rho-\beta+1+1 / \gamma)}
\end{aligned}
$$

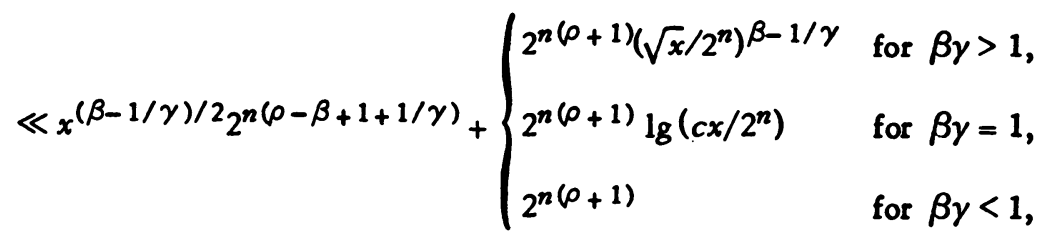


i.e.,

$$
\begin{array}{ll}
T_{n} \ll x^{(\beta-1 / \gamma) / 2} 2^{n(\rho-\beta+1+1 / \gamma)} & \text { for } \beta y>1, \\
T_{n} \ll 2^{n(\rho+1)} \lg x & \text { for } \beta y=1, \\
T_{n} \ll x^{(\beta-1 / \gamma) / 2} 2^{n(\rho-\beta+1,+1 / \gamma)}+2^{n(\rho+1)} & \text { for } \beta y<1 .
\end{array}
$$

First assume that $\rho-\beta+1+1 / \gamma<0$. Then necessarily $\beta y>1$, and if

$$
S=\sum_{x^{1 / 2(\gamma+1)}} T_{\alpha^{n} 2^{n} \sqrt{x}},
$$

then we find by (11) that

$$
S \ll x^{(\beta-1 / \gamma) / 2+(\rho-\beta+1+1 / \gamma) / 2(\gamma+1)}=x^{f} .
$$

Next, let $\rho-\beta+1+1 / \gamma=0$. Then again $\beta y>1$, and by (11) we have

$$
S \ll x^{(\beta-1 / \gamma) / 2} \lg x=x^{(\rho+1) / 2} \lg x .
$$

Finally, let $\rho-\beta+1+1 / \gamma>0$. In this case we sum (11)-(13) over all $n$ with $2^{n} \ll \sqrt{x}$ and obtain

$$
\begin{array}{ll}
s \ll x^{(\rho+1) / 2} & \text { for } \beta y \neq 1, \\
s \ll x^{(\rho+1) / 2} \lg x & \text { for } \beta y=1 .
\end{array}
$$

From (10), (14)-(17) and (8) we obtain the assertions of the Main Theorem.

From our Main Theorem and assertions (A) and (B) of the Introduction, we are able to state the following corollary (cf. Theorem 8 of [G]).

Corollary 1. Let $\gamma$ be the least upper bound of all the numbers $r>0$, for which the inequality $P_{k} \ll k^{-\tau}$ bas infinitely many solutions. Then

$$
\limsup _{x \rightarrow+\infty} \frac{\lg F(x)}{\lg x}=\max \left(\frac{\beta y+\rho}{2(y+1)}, \frac{\rho+1}{2}\right) .
$$

$($ For $\gamma=+\infty$ we put $(\beta \gamma+\rho) / 2(\gamma+1)=\beta / 2$.

Using the inequalities

$$
F_{0, r / 2-1}(x) \leq F_{1}(x) \leq F_{0, r / 2-1}(x) \lg x
$$

and the hypothesis of Corollary 1, we obtain

Cordlary 2. Let $\gamma$ be defined as in Corollary 1. Then 


$$
\limsup _{x \rightarrow+\infty} \frac{\lg F_{1}(x)}{\lg x}=\max \left(\left(\frac{r}{4}-\frac{1}{2}\right) \frac{y}{y+1}, \frac{1}{2}\right) .
$$

Let us recall that moreover

$$
F_{1}(x) \ll x^{r / 4-1 / 2} \sum_{k \leq \sqrt{x}} \frac{\lg 2 k}{k^{r / 2-1}} \ll \begin{cases}x^{r / 4-1 / 2} & \text { for } r>4, \\ \sqrt{x} \lg ^{2} x & \text { for } r=4, \\ \sqrt{x} \lg x & \text { for } r=2,3,\end{cases}
$$

and

$$
F_{1}(x) \gg \sum_{k \leq \sqrt{x}} \lg 2 k \gg \sqrt{x} \lg x .
$$

These results together with the results of [6] thus give full information about "exact order" of the functions $F(x)$ and $F_{1}(x)$.

III. Applications of the Main Theorem. From the Main Theorem and (3)-(5) - we obtain immediately:

Theorem 1. Let $r>4$ and let $\gamma$ denote the least upper bound of all the numbers $\tau>0$, for whicb the inequality $P_{k} \ll k^{-\tau}$ bas infinitely many solutions. Let $0 \leq \rho \leq r / 2-2-1 / \gamma($ for $\gamma=+\infty$ we put $1 / \gamma=0$ and $1 / 2(\gamma+1)=0)$. Then

$$
P_{\rho}(x) \ll x^{r / 2-1-(r / 2-1-\rho) / 2(\gamma+1)+\epsilon}
$$

for every $\epsilon>0$. Let $\rho>r / 2-2-1 / \gamma, \rho \geq 0$. Then

$$
P_{\rho}(x) \ll x^{r / 2+\rho / 2} \lg ^{K} x
$$

where $\kappa=1$ for $\rho=0$ and for $\rho=r / 2-2$, else $\kappa=0$. If $\gamma=+\infty$ and $0 \leq \rho<$ $r / 2-2$ then moreover, $P_{\rho}(x) \ll x^{r / 2-1}$.

Theorem 2. Let $\gamma$ be defined as in Theorem 1. Let $0 \leq \rho \leq r / 2-3 / 2-1 / 2 \gamma$. Tben

$$
M_{\rho}(x) \ll x^{r-1-(r / 2-1-\rho) /(\gamma+1)+\epsilon}
$$

for every $\epsilon>0$. Let $\rho>r / 2-3 / 2-1 / 2 \gamma, \rho \geq 0$. Then

$$
M_{\rho}(x) \ll x^{\gamma / 2+\rho+1 / 2} 1 g^{\kappa} x,
$$

where $\kappa=1$ for $\rho=r / 2-3 / 2$, else $\kappa=0$. If $\gamma=+\infty$ and $0 \leq \rho<r / 2-3 / 2$ then moreover $M_{\rho}(x) \ll x^{r-1}$. 
In papers [5] and [8] the following results are proved ( $\gamma$ is as above):

(D) If $A(x) \equiv 0$, then

$$
P_{\rho}(x)=\Omega\left(x^{(r-1) / 4+\rho / 2}\right) \text { and } M_{\rho}(x) \gg x^{r / 2+\rho+1 / 2} \text {. }
$$

(E) Let $b_{1}=b_{2}=\cdots=b_{r}=0,0 \leq \rho \leq r / 2-1$. Then

$$
\begin{gathered}
P_{\rho}(x)=\Omega\left(x^{r / 2-1-(r / 2-1-\rho) / 2(\gamma+1)-\epsilon}\right) \quad \text { and } \\
M_{\rho}(x)=\Omega\left(x^{r-1-(r / 2-1-\rho) /(\gamma+1)-\epsilon)}\right.
\end{gathered}
$$

for every $\epsilon>0$.

Let us note that the assumption $b_{1}=b_{2}=\cdots=b_{r}=0$ cannot be omitted generally. (See [8, p. 271] or [4, p. 393].) These results together with Theorems 1 and 2 give our final results, which partly solve the basic problem in the theory of lattice points with weight in many dimensional ellipsoids.

Theorem 3. Let $\gamma$ be defined as in Theorem 1 and $b_{1}=b_{2}=\cdots=b_{p}=0$. Let $0 \leq \rho \leq r / 2-2-1 / \gamma$. Then

$$
\limsup _{x \rightarrow+\infty} \frac{\lg \left|P_{\rho}(x)\right|}{\lg x}=r / 2-1-\frac{1}{2(y+1)}(r / 2-1-\rho) .
$$

Theorem 4, Let $\gamma$ be defined as in Theorem 1 and $\kappa$ as in Theorem 2. Let $\rho>r / 2-3 / 2-1 / 2 \gamma$ and $\rho \geq 0$. Then

$$
x^{r / 2+\rho+1 / 2} \ll M_{\rho}(x) \ll x^{r / 2+\rho+1 / 2} \lg ^{\kappa} x .
$$

Let $0 \leq \rho<r / 2-3 / 2-1 / 2 y$ and $b_{1}=b_{2}=\cdots=b_{r}=0$. Then

$$
\underset{x \rightarrow+\infty}{\lim \sup _{x}} \frac{\lg M_{\rho}(x)}{\lg x}=r-1-\frac{1}{\gamma+1}(r / 2-1-\rho) \text {. }
$$

\section{REF ERENCES}

1. B. Diviצ, Über Gitterpunkte in mehrdimensionalen Ellipsoiden. I, Czechoslovak Math. J. 20 (95) (1970), 130-139. MR 41 \#165.

2. G. H. Hardy and J. E. Littlewood, Some problems of Diophantine approximation: The lattice-points of a right-angle triangle, (second memoir), Abh. Math. Sem. Univ. Hamburg 1 (1929), 212-249.

3. B. Novák, Verallgemeinerung eines Peterssonschen Satzes und Gitterpunkte mit Gewichten, Acta Arith. 13 (1967/68), 423-454. MR 37 \#151.

4. - On lattice points with weight in high-dimensional ellipsoids, Acta Arith. 14 (1967/68), 371-397. MR 37 \#6243.

5. - Mean value theorems in the theory of lattice points with weight. II, Comment. Math. Univ. Carolinae 11 (1970), 53-81. MR $41 \# 6807$. 


\section{BŘETISLAV NOVÁK}

6. B. Novak, On a certain sum in number theory, Comment. Math. Univ. Carolinae 12 (1971), 669-685.

7. - Mittelwertsätze der Gitterpunktlehre. II, Časopis P̌st. Mat. 96 (1971), 245-261, 300. (Czech) MR 45 \#191.

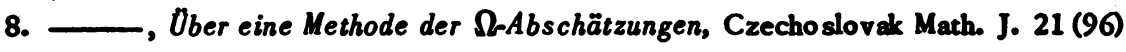
(1971), 257-279. MR 45 \#1843.

9. ——, Uber Gitterpunkte in mehrdimensionalen Ellipsoiden, Czecho slovak Math. J. 22 (97) (1972), 495-507.

DEPARTMENT OF MATHEMATICS, CHARL ES UNIVERSITY, SOKOLOVSKA 83, PRAHA 8, CZECHOSLOVAKIA 